\title{
Visualization of submicron Si-rods by SPR-enhanced total internal reflection microscopy
}

\author{
O.V. Rengevych, G.V. Beketov, Yu.V. Ushenin \\ V. Lashkaryov Institute of Semiconductor Physics, National Academy of Sciences of Ukraine, \\ 45, prospect Nauky, 03028 Kyiv, Ukraine; \\ Phone: +38(044) 525-40-20; e-mail: o.v.shynkarenko@gmail.com
}

\begin{abstract}
The potential of surface plasmon resonance-enhanced total internal reflection microscopy for visualization of submicron particles has been demonstrated using submicron-sized silicon rods as a test object. Submicron Si-rods were deposited onto the surface of a plasmon-supporting gold film by sedimentation from suspension, and their images were obtained using optical microscope with SPR excitation. Quality of images obtained in this way was compared with images viewed from the prism side in the SPR microscopy configuration. Specific features of light scattering from filiform objects are discussed. The study was aimed at development of a novel type of SPR-based biosensor relied upon direct count of biological species of interest (bacteria, viruses, large biomolecular complexes).
\end{abstract}

Keywords: surface plasmon resonance, total internal reflection microscopy, submicron Si-rods, counting biosensor, pathogen detection.

Manuscript received 03.02.14; revised version received 18.07.14; accepted for publication 29.10.14; published online 10.11.14.

\section{Introduction}

Introduction of SPR biosensors more than 2 decades ago provided a strong impact on progress in biochemical science and related disciplines, allowing label-free realtime characterization of biomolecular interactions [1-3]. By now, this technique has also found numerous applications in many other areas, from electrochemical studies to food quality control and environmental monitoring. Growing use of SPR has stimulated research focused on developing new SPR-related techniques: label-enhanced SPR, surface plasmon fluorescence spectroscopy, SPR microscopy (SPRM), also referred to as SPR imaging (SPRI), etc. [4-12]. SPRM was first to demonstrate by B. Rothenhäusler and W. Knoll for imaging of low-contrast samples with high lateral resolution [13]. Currently this method is related mainly to accessing multi-element arrays of macroscopic sensitive areas at the sensor surface and represents just a high-throughput multichannel version of the classical
SPR-biosensor. Recent studies aimed at SPR-assisted visualization of single cells and viruses utilize optical arrangement very similar to that used in SPR imaging [14-16]. General drawback of this technique is strong interference effects arising from light diffraction on particles, which size is comparable with the wavelength. Interference patterns result in splattering of the image, thus hampering true visualization of the particles.

In our study, we demonstrate advantages of alternative approach, based on scattering of electromagnetic field associated with surface plasmons into the external medium. Similar principle lies at the core of the Total Internal Reflection Microscopy (TIRM) [17]. SPR results in enhancement of the evanishing electromagnetic field compared to ordinary TIR, therefore this method can be referred to as an SPRenhanced TIRM. The standard SPR biosensor utilizes a total internal reflection (TIR) prism for optical excitation of surface plasmons (SP) in thin (usually close to $50 \mathrm{~nm}$ ) $\mathrm{Au}$ film, deposited onto surface of a transparent 
substrate. This method of SP excitation is known as the Kretchmann configuration [18]. At a specific angle of incidence, the so-called resonant angle, the intensity of the light reflected from the $\mathrm{Au}$ film, drops down to a minimum due to the resonant excitation of SP. The SPR biosensor exploits dependence of the resonant angle on the adsorption of arbitrary substance on the Au surface. The feasibility of using this effect for biosensing was based on development of functionalization technologies for gold. They allowed to immobilize on the Au surface biomolecules capable of selective binding to specific species (the analyte), which are present in the liquid probe. The output signal of the biosensor is then dependent on the averaged number of specifically bound molecules of the analyte. This method finds the most straightforward applications for label-free immunoassaying [19-22].

Phenomenon underlying the proposed approach consists in scattering of light into the external medium from relatively large particles placed on the surface of the SPR sensor. Surface plasmons are associated with the evanescent electromagnetic field, spreading outside the $\mathrm{Au}$ surface over the distance of $\sim 1 / 4$ of the wavelength. When the surface is microscopically homogeneous, no light is transmitted into the ambience. However, local inhomogeneities with size, comparable to or less than the wavelength, distort the space distribution of the evanescent field, which makes them the effective emitters of light. Exploration of confined electromagnetic fields and their interactions with conduction electrons at metallic interfaces or small metallic nanostructures has become now a subject of the emerged field of nanophotonics and plasmonics [23]. SPR-assisted visualization of small particles by light scattering may appear a promising concept for development of fast, sensitive, and robust method for revealing pathogenic microorganisms and viruses.

\section{Sample preparation and experimental techniques}

Feasibility of visualization of submicron particles located at the surface of the plasmon-supporting metal film by light scattering was investigated using submicron-sized silicon rods as a test object. This choice was made owing to their easily recognizable shape, as well as high index of refraction $(n=3.865)$ and relatively low optical absorption $(k=0.015)$ for $\lambda=$ $0.65 \mu \mathrm{m}$, which were conductive to high intensity of light scattering. The rods were grown by VLS process in a closed volume using the chemical gas-conveying reaction with $\mathrm{Au}$ nanodroplets as a catalyst [24]. This process resulted in both velvet-like coverage formed by Si-rods on the walls of the silica ampoule and the entangled clots of $\mathrm{Si}$ filiform crystals (FC) in the ampoule volume (Fig. 1). To avoid mechanical destruction, the as-grown tangles of $\mathrm{Si}$-rods were disengaged by stirring in ethanol until a homogeneous suspension is formed. Ethanol was found to be the most suitable solvent for this purpose, yielding high enough stability of the suspension without addition of surfactants. The Si-rods were then separated by size using sedimentation method. The diameter of separated Si-rods was evaluated with the scanning probe microscopy (SPM). The submicron fraction (Fig. 2) was then taken for the experiment to mimic the pathogenic microorganisms.

Plasmon-supporting sensor chips were manufactured by thermal evaporation of $\mathrm{Au}$ onto $\mathrm{F} 1$ glass substrates $20 \times 20 \times 1 \mathrm{~mm}$ in size with a $\mathrm{Cr}$ adhesive sublayer. Optimal thickness of $\mathrm{Au}$ film for efficient excitation of surface plasmon resonance is $\sim 50 \mathrm{~nm}$. Submicron Si-rods were deposited onto the Au surface of the sensor chips from the ethanol suspension. Special measures were taken to avoid conglutination of Si-rods during deposition.

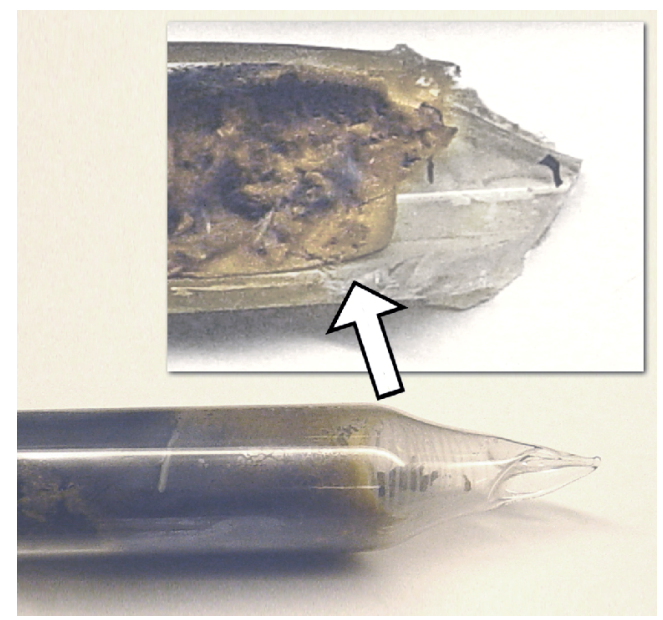

Fig. 1. Growth of submicron Si-rods by using VLS process. Appearance of as-grown clots of Si filiform crystals in the unsealed ampoule (above).

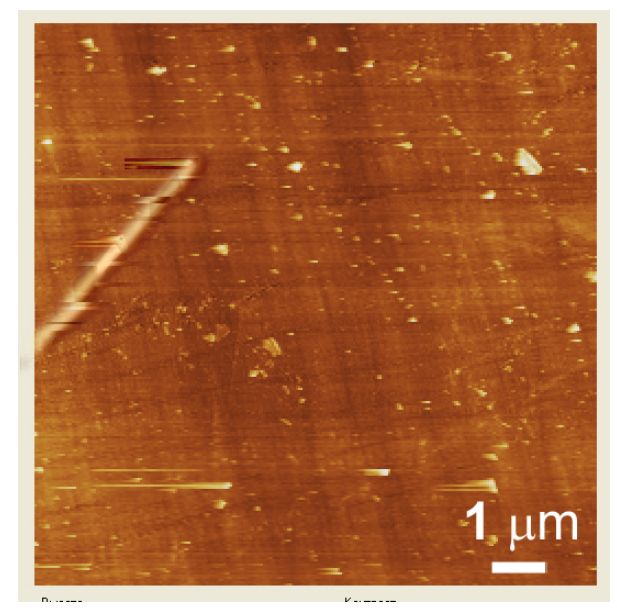

Fig. 2. AFM image of typical submicron Si-rod after separation by size using the sedimentation method. 


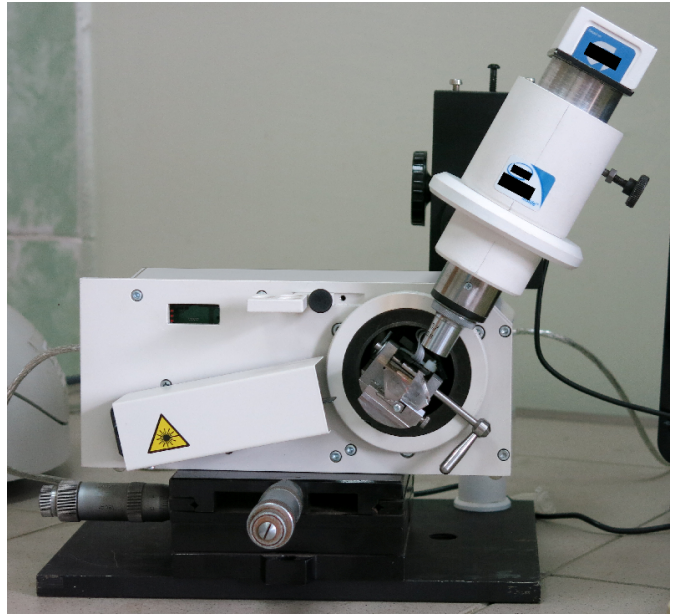

Fig. 3. Experimental setup for visualization of submicron particles by SPR-enhanced total internal reflection microscopy.

Microscopic images of Si-rods deposited onto $\mathrm{Au}$ layer were obtained using custom-built experimental setup based on the model Plasmon-6 biosensor (ISP NASU, Ukraine) [25] (Fig. 3). The optical layout of the setup is shown in Fig. 4. The Kretchmann configuration was used for optical excitation of SPR with the 3-mW GaInP/AlGaInP semiconductor laser diode $(\lambda=650 \mathrm{~nm})$ serving as a light source and a total internal reflection (TIR) prism as a coupler. The laser radiation was focused into $5 \times 1 \mathrm{~mm}$ spot. Measurements were carried out in air environment with TIR prism made from K8 (BK7) optical glass. Specific features of images retrieved using scattered and reflected light beams have been compared for different orientations of the substrate.

\section{Experimental results and discussion}

For K8 glass prism $(n=1.5141$ at $\lambda=0.65 \mu \mathrm{m})$ and $\mathrm{Au}$ thickness $45 \mathrm{~nm}$, the calculated value of the resonant angle, $\varphi_{r}$, is $43.368^{\circ}$. For calculation, the optical constants of $\mathrm{Au}$ were assumed to be $0.2+i \cdot 3.8$. Precise positioning of the prism at the resonant angle was made under control of both the intensity of the reflected beam and the visual brightness of the specimen surface. Presence of the nanorods at the Au/air interface results in breaking the momentum conservation for surface plasmons, thus letting the electromagnetic waves to be emitted into the external medium (air). The scattered light was focused by the objective lens onto the solidstate imaging device, and the images were acquired using standard software. All the images, except that shown in Fig. 7a, were obtained using the objective lens with numerical aperture 0.11 .

Typical images of Si-rods are shown in Fig. 5. The Si-rods look like bright touches on a dark background, whose length generally does not exceed $100 \mu \mathrm{m}$. Ideally, at the resonance angle no radiation should be transmitted into the air through the $\mathrm{Au}$ layer. Nevertheless, numerous luminous spots and faintly glowing nebulas can also be observed in the background. This radiation was attributed to initially existing surface defects resulted from contamination or imperfect technology. These defects can clearly be seen in Fig. 2. Noteworthy is pronounced difference in scattering intensity between the rods oriented in a direction perpendicular to the direction of SPs propagation, and the other randomly oriented rods. In principle, the scattering intensity can be influenced by several factors. In particular, in case of evanescent field, it is dependent on the distance between the scattering particle and the surface [17]. To ascertain that scattering is really orientation-dependent, images of the same Si-rod acquired at different angular orientations were compared (Figs $6 \mathrm{a}$ and $6 \mathrm{~b}$ ). It is clearly seen that for the Si-rod the angular deviation of only $\sim 10^{\circ}$ causes drastic drop of light scattering intensity, while brightness of dot-like scatterers remains practically unchanged. Under conditions of directional illumination, strong dependence of visibility for long, thin objects on the direction and polarization of the incident light was also observed in regular light microscopy [26]. Theoretical analysis of this effect shows that intensity of light scattering by infinite homogeneous cylinders is a function of illumination angle, polarization of the incident light, and the angle of observation [27]. Though this analysis is not directly applicable to the scattering of light by the particle interacting with the evanescent field, dependence of intensity on the observation angle can be conjectured also for this case. To verify this assumption, images of the same area of the surface were obtained using objective lenses with different numerical aperture (Figs $7 \mathrm{a}$ and $7 \mathrm{~b}$ ). The image obtained with the numerical aperture 0.4 evidently contains more details than that obtained with the numerical aperture 0.11 . This is in agreement with the above assumption, because larger aperture admits larger angular cone of the scattered light. Nevertheless, this does not rule out the existence of other physical effects influencing the visible brightness of the rods in dependence of their orientation. Thus, considering the rod as a limiting case of a thin strip of transparent material at the Au surface, it could be surmised that the total internal reflection also contributes to this effect. It is evident that a wide strip oriented along the direction of SPs propagation, will not transfer light into the external medium, when its angle of incidence exceeds the critical one. Unfortunately, this possibility could not be explored with the experimental setup used in this study and will be the subject of further researches.

The image of the sample obtained in the SPRM configuration is presented in Fig. 8. The contrast of this picture is much lower in comparison with the images obtained with the SPR-enhanced TIRM, though no dependence of brightness of Si-rods on orientation can be observed. Highly heterogeneous brightness of the background also hampers visualization of the point-like scatterers, which are clearly resolved with the use of the SPR-enhances TIRM arrangement. 


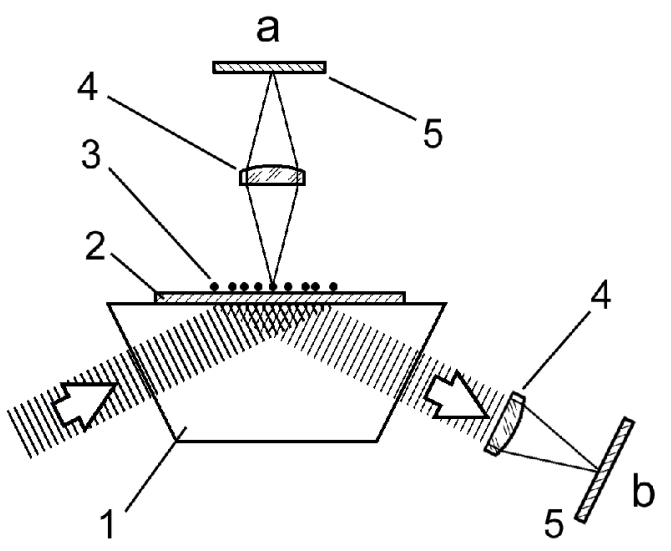

Fig. 4. Layout of optical configuration employed for visualization of submicron particles by SPR-enhanced total internal reflection microscopy $(a)$ and surface plasmon resonance microscopy $(b): 1$ - total internal reflection prism, 2 - plasmon-supporting $\mathrm{Au}$ layer, 3 - test objects, 4microscope lens, 5 - solid-state focal plane.

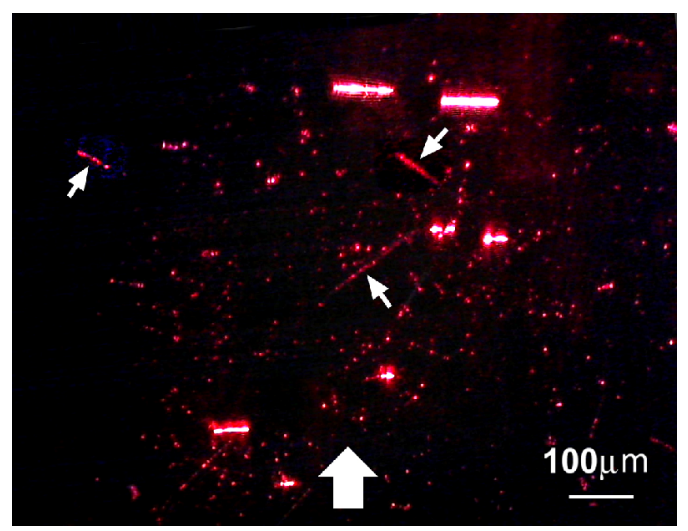

Fig. 5. Typical images of Si-rods obtained by the SPRenhanced total internal reflection microscopy. The thick arrow shows direction of SPs propagation; small arrows indicate weak images of Si-rods misoriented against the perpendicular to this direction.

The experimental results presented above demonstrate advantages of the SPR-enhanced TIRM for imaging of submicron objects located at the surface of the plasmon-supporting film. This method can find applications in biosensor technology for detection of trace amounts of infectious microorganisms in various media. Feasibility of pathogens detection with the SPRbased biosensors has already been demonstrated [28-31]. The image quality obtained with the SPR-enhanced TIRM was sufficient for counting the scattering particles of submicron size. Transfer of counting methods to biosensor technology may result in considerable improvement of sensitivity, as compared to measuring the integral effect of specific binding exploited in current SPR-based biosensors.

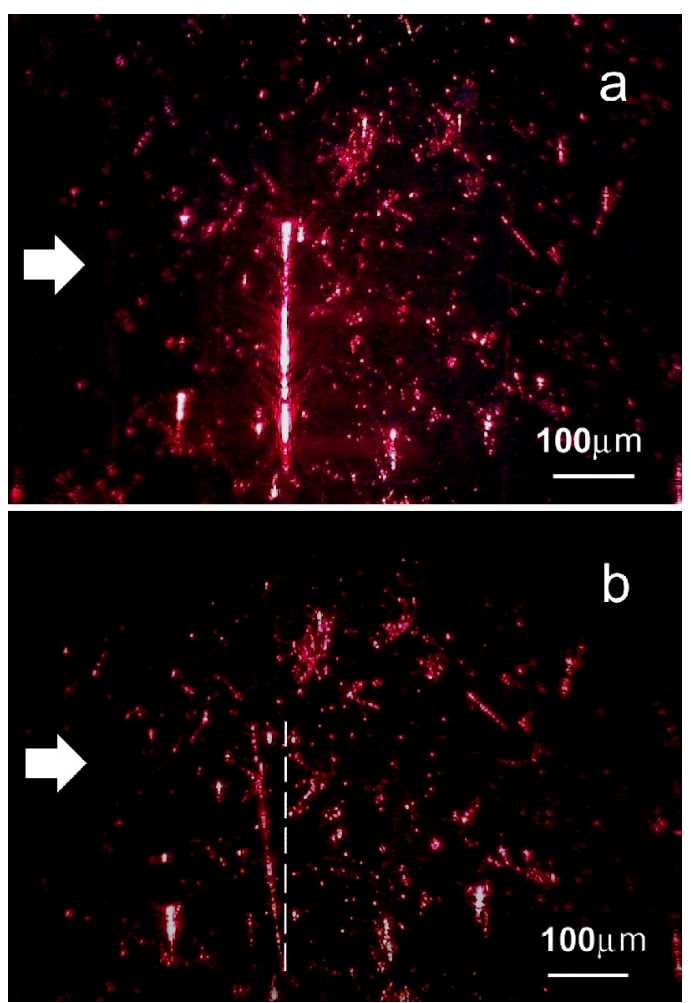

Fig. 6. Images of Si-rod obtained by the SPR-enhanced total internal reflection microscopy with different orientations against the perpendicular to the direction of SPs propagation.
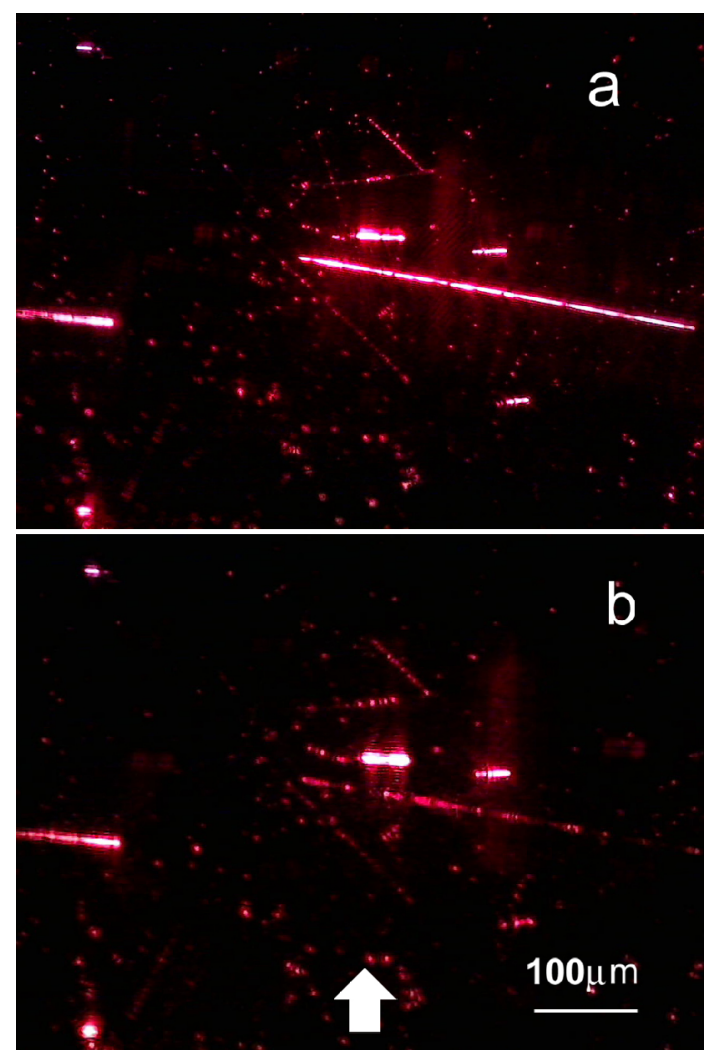

Fig. 7. Images of Si-rod obtained using objective lenses with numerical aperture $0.4(a)$ and $0.11(b)$. 


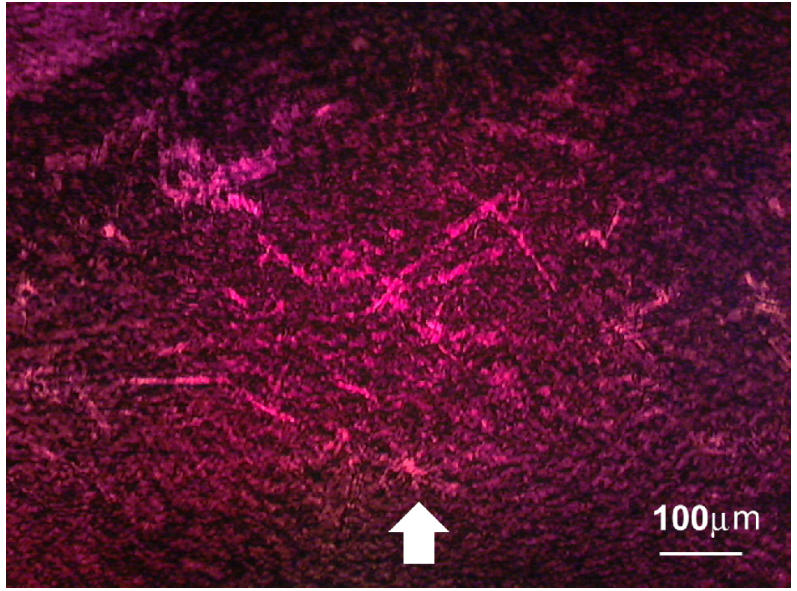

Fig. 8. Typical images of Si-rods obtained using surface plasmon resonance microscopy configuration.

\section{Conclusions}

Advantages of the novel method, the SPR-enhanced TIRM, for imaging of submicron particles immobilized on the surface of the plasmon-supporting film over the surface plasmon resonance microscopy has been demonstrated. Usage of submicron-sized silicon rods as a test object allowed for clear distinguishing their images from extraneous defects usually present at the surface.

The pronounced difference in scattering intensity between the rods oriented in the direction perpendicular to that of SPs propagation and other randomly oriented rods have been observed. Experimental evidence was obtained that this behaviour can be partially attributed to angular dependence of the scattered intensity on the observation angle. Other possible mechanisms of this dependence have been also discussed.

This study was aimed at development of a novel type of SPR-based technique relied upon direct count of biological species of interest (bacteria, viruses, large biomolecular complexes), rather then measuring the integral effect of specific binding exploited in current SPR-based biosensors.

The search for innovative principles of nanodiagnostic assays for detection of infectious pathogens is an active area of investigation. It is expected that the proposed method could find applications in biochemistry, biomedicine, food safety inspection, and environmental monitoring, especially for fast detection and identification of trace amounts of pathogens in the natural water resources.

\section{Acknowledgements}

The authors would like to extend their most sincere appreciation to Dr. Prof. A. Klimovskaya for providing the submicron-sized silicon nanorods for this study.

This work was partially supported by Swiss National Science Foundation through SCOPES JRP IZ73Z0_152661.

\section{References}

1. C. Hahnefeld, S. Drewianka, F.W. Herberg, Determination of kinetic data using surface plasmon resonance biosensors, Chap. 19 in: Molecular Diagnosis of Infectious Diseases, Eds. J. Decler and U. Reischl, p. 299-320, Humana Press, series Methods in Molecular Medicine, 94 (2004).

2. N.J. de Mol, M.J.E. Fisher, Kinetic and thermodynamic analysis of ligand-receptor interactions: SPR applications in drug development, Chap. 5 in: Handbook of Surface Plasmon Resonance, Eds. R.B.M. Schasfoort and A.J Tudos, p. 123-172, RSC Publishing (2008).

3. R.J. Green, R.A. Frazier, K.M. Shakeshe, M.C. Davies, C.J. Roberts, S.J.B. Tendler, Surface plasmon resonance analysis of dynamic biological interactions with biomaterials // Biomaterials, 21 p. 1823-1835 (2000).

4. N. Granqvist, A. Hanning, L. Eng, J. Tuppurainen, T. Viitala, Label-enhanced surface plasmon resonance: A new concept for improved performance in optical biosensor analysis // Sensors, 13, p. 15348-15363 (2013).

5. E. Hutter, J.H. Fendler, D. Roy, Surface plasmon resonance studies of gold and silver nanoparticles linked to gold and silver substrates by 2aminoethanethiol and 1,6-hexanedithiol // J. Phys. Chem. B, 105(45), p. 11159-11168 (2001).

6. Zhong-Shu Chang, Chi-Yuan Chang, Rong-Seng Chang, Surface plasmon resonance design of chiptype sensor with nanoparticles // Intern. J. Innovative Computing, Information and Control, 9(10) p. 3955-3964 (2013).

7. K.M. Byun, N.-H. Kim, J.W. Leem, J.S. Yu, Enhanced surface plasmon resonance detection using porous ITO-gold hybrid substrates // Appl. Phys. B, 107, p. 803-808 (2012).

8. W. Knoll, A. Kasry, Jing Liu, T. Neumann, Lifang Niu, H. Park, H. Paulsen, R. Robelek, Danfeng Yao and Fang $\mathrm{Yu}$, Surface plasmon fluorescence techniques for bioaffinity studies, Chap. 9 in: Handbook of Surface Plasmon Resonance, Eds. R.B.M. Schasfoort and A.J. Tudos, p. 275-312, RSC Publishing (2008).

9. A.J. Thiel, A.G. Frutos, C.E. Jordan, R.M. Corn, L.M. Smith, In situ surface plasmon resonance imaging detection of DNA hybridization to oligonucleotide arrays on gold surfaces // Anal. Chem. 69, p. 4948-4956 (1997)/

10. C.T. Campbell, G. Kim, SPR microscopy and its applications to high-throughput analyses of biomolecular binding events and their kinetics // Biomaterials, 28 p. 2380-2392 (2007).

11. E. Fu, T. Chinowsky, K. Nelson, P. Yager, SPR imaging for clinical diagnostics, Chap. 10 in: Handbook of Surface Plasmon Resonance, Eds. R.B.M. Schasfoort and A.J. Tudos, p. 313-332, RSC Publishing (2008). 
12. L. Malic, Bo Cui, M. Tabrizian, T. Veres, Nanoimprinted plastic substrates for enhanced surface plasmon resonance imaging detection // Opt. Exp. 17(22), p. 20386-20392 (2009).

13. B. Rothenhäusle, W. Knoll, Surface-plasmon microscopy // Nature, 332, p. 615-617 (1988).

14. A. Zybin, Y.A. Kuritsyn, E.L. Gurevich, V.V. Temchura, K. Überla, K. Niemax, Surface plasmon resonance for detection of dielectric nanoparticles and viruses // Plasmonics, 5, p. 31-35 (2010).

15. E.L. Gurevich, V.V. Temchura, K. Überla, A. Zybin, Analytical features of particle counting sensor based on plasmon assisted microscopy of nano objects // Sensors and Actuators B, 160, p. 1210-1215 (2011).

16. V. Shpacovitch, Application of surface plasmon resonance (SPR) for the detection of single viruses and single biological nano-objects // J. Bacteriol. Parasitol. 3(7), e110, 3 p. (2012).

17. D.C. Prieve, F. Luo, F. Lanni. Brownian motion of a hydrosol particle in a colloidal force field // Faraday Discussions of the Chemical Society, 83, p. 297-307 (1987).

18. H. Raether, Surface Plasmons on Smooth and Rough Surfaces and on Gratings. Springer Tracts in Modern Physics, Vol. 111, Springer Berlin, 1988.

19. A. Kausaite, M. van Dijk, J. Castrop, J.P. Baltrus, A. Ramanaviciene, J. Acaite, A. Ramanavicius, Surface plasmon resonance label-free monitoring of antibody antigen interactions in real time // Biochemistry and molecular biology education, 35(1), p. 57-63 (2007).

20. J.A. Lofgren, S. Dhandapani, J.J. Pennucci, C.M. Abbott, D.T. Mytych, A. Kaliyaperumal, S.J. Swanson, M.C. Mullenix, Comparing ELISA and surface plasmon resonance for assessing clinical immunogenicity of panitumumab // J. Immunol. 178, p. 7467-7472 (2007).

21. T. Candresse, H. Lot, S. German-Retana, R. Krause-Sakate, J. Thomas, S. Souche, T. Delaunay, M. Lanneau, O. Le Gall, Analysis of the serological variability of Lettuce mosaic virus using monoclonal antibodies and surface plasmon resonance technology // J. Gen. Virol. 88, p. 26052610 (2007).
22. P. Singh, T. Onodera, Y. Mizuta, K. Matsumoto, N. Miura, K. Toko, Novel DNP-KLH protein conjugate surface for sensitive detection of TNT on SPR immunosensor // Sensor. Mater. 19, p. 261273 (2007).

23. S.A. Maier, Plasmonics: Fundamentals and Applications. Springer Science+Business Media LLC (2007).

24. Heon-Jin Choi, Vapor-Liquid-Solid growth of semiconductor nanowires, Chap. 14 in: Semiconductor Nanostructures for Optoelectronic Devices, Ed. Gyu-Chul Yi, p. 1-36, SpringerVerlag Berlin Heidelberg (2012).

25. "Plasmon" line of Surface Plasmon Resonance spectrometers from Surface Plasmon Resonance Spectrometry Group of ISP NASU, http://www. plasmon.org.ua/PRODUCTS.HTM

26. R. Arimoto, J.M. Murrayt, Orientation-dependent visibility of long thin objects in polarization-based microscopy // Biophys. J. 70(6), p. 2969-2980 (1996).

27. C.F. Bohren, D.R. Huffman, Angular dependence of scattering, Chap. 13 in: Absorption and Scattering of Light by Small Particles, p. 321-429. John Wiley, New York, 1998.

28. P. Tallury, A. Malhotra, L.M. Byrne., S. Santra, Nanobioimaging and sensing of infectious diseases // Adv. Drug Delivery Rev. 62, p. 424-437 (2010).

29. A.A. Yanik, Min Huang, O. Kamohara, A. Artar, T.W. Geisbert, J.H. Connor, H. Altug, An optofluidic nanoplasmonic biosensor for direct detection of live viruses from biological media // Nano Lett. 10(12), p. $4962-4969$ (2010).

30. B. Barlen, S.D. Mazumdar, O. Lezrich, P. Kämpfer, M. Keusgen, Detection of Salmonella by surface plasmon resonance // Sensors, 7, p. 1427-1446 (2007).

31. Jing $\mathrm{Xu}$, Jia-yu Wan, Song-tao Yang, Shou-feng Zhang, Na Xu, Nan Li, Ji-ping Li, Hai-ying Wang, Xue Bai, Wen-sen Liu, A surface plasmon resonance biosensor for direct detection of the rabies virus // Acta Veterinaria Brno, 81, p. 107111(2012). 